\section{The NeuroVascular Coalition: Birth, Death, and Endovascular Stroke Training}

The paper "Performance and Training Standards for Endovascular Ischemic Stroke Treatment" ${ }^{1}$ and the accompanying editorial ${ }^{2}$ both make the claim that this training standard was approved by the NeuroVascular Coalition. ${ }^{3}$ As Founder, first Co-Chair, creator of the name and the abbreviation (NVC), and owner of the Web address (www.neurovascularcoalition.org), I would like to correct important facts and clarify misconceptions.

The creation of the NeuroVascular Coalition in 2003-2004 was during the early days of carotid stent placement. The 4500-member Society of Interventional Radiology (SIR) and the 3000-member American Society of Neuroradiology (ASNR) were the cornerstones of the NeuroVascular Coalition for 2 reasons. First, almost all cerebral angiographic examinations in the United States were performed and/or imaged by our members (thus pertinent to carotid stent placement). We (American Society of Interventional and Therapeutic Neuroradiology [ASITN], SIR, ASNR) all have at least 6 months of formal Accreditation Council for Graduate Medical Educationdefined training in the neurosciences (neuroanatomy, neuropathology, neuro-CT, MR imaging, neuroangiography, single-photon emission CT, carotid Doppler, and so forth), and our members invented the field of "Interventional Neuroradiology," thus the name. The founding societies of NVC were ASITN, ASNR, SIR, the American Academy of Neurology, the Congress of Neurological Surgeons, and the American Association of Neurological Surgeons (and the combined Cerebrovascular Section of the 2 neurosurgery societies). ${ }^{3}$ The second reason I created the NeuroVascular Coalition was to give my small society (ASITN/Society of NeuroInterventional Surgery [SNIS]) some clout by joining forces with a team of large and powerful radiologic societies (ASNR and SIR) and then uniting with all the neuroscience societies to speak with 1 authoritative voice. This worked. I, and thus ASITN, became the leader of a new powerful multisociety group: the NeuroVascular Coalition.

Previously, in 2001, the ASITN/SNIS, ASNR, and SIR published an official joint statement, "Emergency Interventional Stroke Therapy," specifically stating that, with appropriate training, physicians from our specialties could treat strokes. ${ }^{4}$ The Meyers et al document ${ }^{1}$ expressly contradicts this prior official document and states that the only people qualified to treat a stroke (as of 2012) are fellowshiptrained neurointerventionists, thus excluding most SIR and ASNR members and fellows. By formal agreement, all NVC decisions must be unanimous. Despite the statement within the "Performance and Training Standards for Endovascular Ischemic Stroke Treatment"1 that the training standards were written and approved by the NeuroVascular Coalition, it was not endorsed by either ASNR or SIR and thus is not an NVC document. SIR and ASNR did not sign the Meyers et al document ${ }^{1}$ for multiple reasons that have been described in depth, ${ }^{5}$ and below. Insistence on a position that excludes most members of ASNR and SIR ultimately splintered the NVC and, in addition to being futile, was, in my humble opinion, a distressing strategic faux pas.

The Meyers et al document ${ }^{1}$ might also exclude many of the fellows/members of the authoring societies. The requirement that a neurointerventional fellow must be the "primary operator" for 10 complete stroke cases during their fellowship is laudable but seldom achieved; there are very few neurointerventional fellowships in the

Indicates open access to non-subscribers at www.ajnr.org
United States that provide enough experience. Most fellows would be fortunate to even see 10 endovascular stroke cases, yet neurointerventional fellowship directors must now personally certify all fellows as being the "primary operator" on at least 10 cases.

I believe that intra-arterial stroke therapy is effective but that it requires many more well-trained physicians to provide this service to the United States. I agree with Meyers et $\mathrm{al}^{1}$ and other societies that adequate training is essential for physicians who perform intra-arterial stroke revascularization. However, I disagree that adequate training can only be acquired in a full neurointerventional fellowship (or that such fellowships necessarily provide adequate training), and I disagree that such a requirement will then supply sufficient numbers to adequately staff stroke programs nationwide. Even now, 4000 cardiologists are not enough to provide nationwide endovascular treatment for acute myocardial infarction. There are many neurointerventionists who do not wish to ever be called in the middle of the night, nor can a 1- to 2-person group do this alone. Many major cities do not currently have a $24 / 7 / 365$ endovascular stroke program. The largest program in the Southeastern United States is in Chattanooga, Tennessee and the largest published single-center series comes from Oklahoma City, Oklahoma. ${ }^{6}$ Of nearly a dozen physicians running these programs, there is only 1 fellowship-trained neurointerventionist.

SIR, with leadership from multiple neurointerventional fellowship-trained physicians who are members of ASNR, SIR, and SNIS (including myself), has published a different training guidelines document for physicians who treat acute ischemic stroke. ${ }^{7}$ In the SIR document, the fundamental premise is that the physician must acquire and demonstrate mastery of the necessary knowledge and skills and confirm this by tracking outcomes. One route is through a good neurointerventional fellowship. Other routes are through training, experience, course work (such as the intensive SIR-sponsored Catheter Lysis of Thromboembolic Stroke course), ${ }^{8}$ proctorship, and examination.

Patient outcomes are the bottom line. In 2001, in association with SNIS, I created the INterventional Stroke Therapy Outcomes Registry (INSTOR: www.strokeregistry.org) to track outcomes of endovascular stroke therapy. Interventional radiologists have entered most cases since then. Submission of cases to a national registry is required by the SIR training standards and by the Meyers et $\mathrm{al}^{1}$ document. I remain hopeful that physicians treating stroke will be concerned about quality improvement and clinical outcomes and will use INSTOR. In that way, we could more powerfully document the quality of care and demonstrate that endovascular stroke therapy works. We would also be better able to focus on patient outcomes rather than on the pedigree of a fellowship.

\section{References}

1. Meyers PM, Schumacher HC, Alexander MJ, et al, for Writing Group for the American Academy of Neurology; AANS/CNS Cerebrovascular Section; Society of NeuroInterventional Surgery; Society of Vascular \& Interventional Neurology. Performance and training standards for endovascular ischemic stroke treatment. AJNR Am J Neuroradiol 2010;31:E8-11

2. Cloft HJ. Interventional Standards. AJNR Am J Neuroradiol 2010;31:3-4

3. Connors JJ, Sacks D, Furlan AJ, et al, for the NeuroVascular Coalition Writing Group. Training, competency, and credentialing standards for diagnostic cervicocerebral angiography, carotid stenting, and cerebrovascular intervention. AJNR Am J Neuroradiol 2004;25:1732-41

4. Emergency interventional stroke therapy: a statement from the American Society of Interventional and Therapeutic Neuroradiology Stroke Task Force of the American Society of Neuroradiology and the Society of Cardiovascular and Interventional Radiology. AJNR Am J Neuroradiol 2001;22:54 
5. Sacks D, Connors JJ III. Commentary: intraarterial stroke revascularization training guidelines. J Vasc Interv Radiol 2009;20:1523-26

6. Belisle JG, McCollom VE, Tytle TL, et al. Intraarterial therapy for acute ischemic strokes. J Vasc Interv Radiol 2009;20:327-33. Epub 2009 Jan 21

7. Connors JJ III, Sacks D, Black CM, et al. Training guidelines for intra-arterial catheter-directed treatment of acute ischemic stroke: a statement from a special writing group of the Society of Interventional Radiology. J Vasc Interv Radiol 2009;20:1507-22
8. http://www.sirweb.org/meetings/SocietyMeetings.shtml. Accessed April 12, 2010

B. Connors

Department of Radiology Vanderbilt University Medical Center Nashville, Tennessee

DOI 10.3174/ajnr.A2175 